\title{
De la « crise du français » à la rénovation de l'enseignement de la langue maternelle : Transformation des contenus théoriques et didactiques dans les manuels de grammaire genevois (1916-1979)
}

\author{
Darme, Anouk \\ Faculté de Psychologie et des Sciences de l'Education (FPSE) - Université de Genève \\ anouk.darme@unige.ch \\ Schneuwly, Bernard \\ Faculté de Psychologie et des Sciences de l'Education - Université de Genève \\ bernard.schneuwly@unige.ch
}

\section{Introduction}

L'instauration de l'instruction primaire publique dans nombre de pays européens au XIX ${ }^{\mathrm{e}}$ siècle s'est accompagnée de l'émergence de disciplines scolaires (Dorier, Leutenegger \& Schneuwly, 2013). Dans ce contexte, le français langue maternelle, issu de composantes diverses, se stabilise petit à petit et aboutit au tournant $\mathrm{du} \mathrm{XX}^{\mathrm{e}}$ à la «première fondation du français » (Savatovsky, 2011, p. 125). On peut dès lors considérer qu'au début du $\mathrm{XX}^{\mathrm{e}}$ siècle, la discipline «Français langue maternelle » est stabilisée et que l'enseignement grammatical y tient une place importante. Or, à la même période, ce dernier est sujet à des critiques et remises en question aussi bien sur le plan théorique que didactique, critiques que nous illustrerons avec les propos de Ferdinand Brunot, ardent défenseur de la réforme de l'enseignement grammatical en France :

\begin{abstract}
[...] S'il y a une crise du français, ce n'est pas que l'on enseigne trop peu la grammaire, c'est qu'on l'enseigne mal : abstractions incompréhensibles, définitions prétentieuses et néanmoins le plus souvent vides, règles fausses, énumérations indigestes, il n'y a qu'à feuilleter quelques pages d'un manuel pour trouver des spécimens variés de ces fautes contre la raison, la vérité et la pédagogie. (Brunot, 1908, cité par Fournier, 1998)
\end{abstract}

Cette « crise du français », comme l'appelle Brunot, et quelques années après lui Charles Bally en Suisse romande ${ }^{1}$, est marquée par une critique sévère des théories et méthodes grammaticales alors en usage à l'école primaire. Issues des principes de la grammaire logique de Port-Royal, elles sont caractérisées sur le plan théorique par une forte influence des procédés d'analyse de la logique et sur le plan didactique, par un enseignement déductif basé sur l'apprentissage par cœur puis l'application de règles grammaticales (Chervel, 1977, 2006). Les propositions élaborées par le courant de remise en question ne se généralisent cependant pas. Il faudra attendre les années 60-70 pour observer une rénovation plus radicale et son institutionnalisation plus générale, comme le note par exemple Bronckart (2001) :

\begin{abstract}
La rénovation engagée en Suisse romande a comme finalité primordiale de mettre en œuvre les propositions de renouvellement des méthodes et des attitudes pédagogiques formulées dès le premier quart du siècle par les tenants de l'Ecole active et de l'Education nouvelle. (p.3)
\end{abstract}

Nous nous proposons d'observer cette évolution générale à travers une étude de cas qui permet de suivre de manière particulièrement claire l'évolution esquissée : celui du canton de Genève. Ce cas se caractérise par deux phénomènes: les moyens d'enseignement y sont obligatoires, élaborés par des instances désignées par l'Etat; le mouvement de l'Education nouvelle y est particulièrement fort, représenté par l'Institut Jean-Jacques Rousseau qui collabore avec des linguistes (Bally, Sechehaye), et qui est en étroite relation avec les associations professionnelles (Hofstetter, Schneuwly, Lussi \& Haenggeli-Jenni, 2006). 
Notre analyse portera sur un corpus composé de trois manuels scolaires, sélectionnés dans la mesure où ils illustrent trois périodes de l'enseignement grammatical dans le canton choisi : le premier, le Cours de langue française (Vignier, 1916) est produit dans les premiers temps de la «crise du français » et se positionne du côté du renouvellement de l'enseignement grammatical; le deuxième, J'apprends la grammaire (Atzenwiler, 1933), coïncide avec l'essor de l'Education nouvelle dans le canton de Genève ; enfin, le dernier, Maîtrise du français (Besson, Genoud, Lipp \& Nussbaum, 1979) présente la spécificité d'être le premier ouvrage de la rénovation de l'enseignement de la langue maternelle en Suisse romande. Il ne s'agit donc pas d'une analyse exhaustive d'un corpus de manuels mais d'une étude menée sur un échantillon représentatif de l'état de l'enseignement de la grammaire à différentes périodes. Notre communication prendra la forme suivante: après une présentation des éléments théoriques servant de cadre à notre réflexion d'une part et de notre méthodologie de recherche d'autre part, nous exposerons les résultats de notre analyse afin d'en esquisser les contenus notionnels et les caractéristiques didactiques ${ }^{2}$.

\section{Cadre théorique, problématique et méthode}

\subsection{Eléments de théorie}

Comme le soulignent Schneuwly et Dolz (2009), le terme grammaire peut recouvrir différentes significations :

[...] Il désigne tour à tour une propriété de toute langue, une discipline scientifique, une branche de la linguistique, un ensemble de symboles reliés entre eux par un ensemble ordonné de règles, l'art d'écrire et de parler correctement, une activité scolaire, des produits manufacturés et commercialisés. (p. 52)

Devant la multiplicité d'acceptions que revêt ce terme, il importe de circonscrire notre objet de recherche. Cette communication s'intéresse donc plus particulièrement à l'évolution de la grammaire scolaire, que nous définirons à l'aide de Chervel (1977), soit «l'ensemble des « connaissances » grammaticales que l'école livre à son public dans son programme d'étude de la langue française » (p. 26).

Par ailleurs, notre analyse s'appuie sur un corpus composé de manuels scolaires. Selon Choppin (1980), le manuel est à la fois un objet, le «support d'un contenu éducatif», un instrument pédagogique et le «véhicule d'un système de valeurs, d'une idéologie, d'une culture » (p. 1). Il sera analysé ici du point de vue de son contenu éducatif et comme instrument didactique et pédagogique. Il conviendra donc de prendre en compte le contexte de production de chaque manuel, que nous esquissons brièvement :

- Le premier manuel de notre corpus s'inscrit dans le contexte de la première remise en question de la grammaire scolaire au tournant du siècle ;

- Le deuxième concorde avec l'apparition du mouvement de l'Éducation nouvelle questionnant le rôle, le statut et, en lien avec la linguistique, les contenus de la grammaire ;

- Enfin, Maîtrise du français (1979) voit le jour dans un contexte de rénovation profonde de l'enseignement du français, à replacer dans un contexte plus général de la «massification du secondaire » et de la révision de tous les plans d'études.

Notre analyse des contenus est donc guidée par un point de vue clairement défini : nous envisageons ces manuels comme des "actualisations» spécifiques des savoirs et démarches d'enseignement de leur époque. En effet, l'adoption d'une nouvelle grammaire par les autorités cantonales résulte d'influences de natures diverses, dont nous dégageons au moins trois types : des facteurs sociaux externes, influençant l'école; des conceptions pédagogiques (activité de l'élève) et des facteurs scientifiques (nouvelles approches académiques). Ce dernier facteur se manifeste sous la forme de ce que l'on appelle la transposition didactique.

Au regard de ce concept, le savoir scolaire se construit au cours d'un processus complexe caractérisé par une mutation du savoir de son milieu d'origine vers le milieu scolaire, où il devient savoir à enseigner 
(transposition externe) puis objet d'enseignement et d'apprentissage (transposition interne) (Chevallard, 1985; Schneuwly \& Dolz, 2009). Au cours de ce processus, le savoir subit deux transformations majeures : pour pouvoir être enseigné, il doit être découpé en unités plus petites, il est ainsi «élémenté »; ensuite, lors de son passage dans le milieu scolaire, le savoir change de sens : à l'origine «savoir utile dans les situations », il devient « savoir à enseigner, savoir à savoir, savoir enseigné » (Schneuwly, 1995, p. 49). Le concept de transposition servant de cadre à notre analyse, un intérêt particulier sera donc porté aux théories de références mobilisées dans les différents ouvrages et à la manière dont elles sont exploitées.

\subsection{Questions de recherche}

A partir de l'axe général de la recherche présenté en introduction et des éléments théoriques exposés précédemment, nous allons donc, à travers une étude de cas et en fonction de la périodisation esquissée plus haut (voir supra 1 et 2.1), analyser les transformations des finalités, contenus et modes d'enseignement de la grammaire dans le canton de Genève. Un ensemble de questions servira de fil conducteur à notre recherche :

- Comment l'appareil notionnel d'une part, la démarche d'enseignement et la conception de l'apprentissage d'autre part se transforment-ils dans le temps? Sous l'influence de quel(s) facteur(s) ?

- Quelle(s) finalité(s) sous-tend(ent) l'enseignement grammatical ? Comment évolue(nt)-elle(s) dans le processus historique?

- Quelles références théoriques sont mobilisées par les auteurs de manuels et quel(s) impact(s) ont-elles sur la structure notionnelle de la grammaire ? Et sur la démarche didactique?

\subsection{Méthode}

Pour répondre à ces questions, nous avons dans un premier temps constitué un corpus de manuels, puis élaboré une méthode d'analyse des manuels retenus.

\subsubsection{Présentation du corpus de manuels}

Le choix des manuels composant le corpus étudié résulte du croisement de plusieurs indices permettant d'attester de leur utilisation. Ces indices sont de trois ordres: l'adoption de ces ouvrages par le Département de l'instruction publique (DIP) ou par la Commission Romande des Moyens d'Enseignement (COROME) pour la période après 1970, caractérisée par une harmonisation de l'enseignement au niveau de la Suisse romande ; la mention dans les programmes ou plans d'études; la présence dans les listes de manuels éditées chaque année par le DIP du canton de Genève et diffusées dans les écoles primaires. Le croisement de ces différents indices nous a donc permis de dégager trois ouvrages dont la durée d'utilisation varie entre vingt et trente ans. Nous avons délibérément écarté les rééditions ou réadaptations (c'est par exemple le cas de la grammaire d'Atzenwiler (1933) qui sera rééditée par le DIP en $1949^{3}$ ), qui feront l'objet de développements ultérieurs. La méthode d'élaboration du corpus ayant été éclaircie, nous allons à présent décrire brièvement les caractéristiques principales des manuels sélectionnés.

Les trois ouvrages sélectionnés sont des moyens d'enseignement officiels dans la mesure où ils ont été adoptés par le Département de l'Instruction publique du canton de Genève. De par leur caractère obligatoire, ils permettent donc de dégager assez justement les savoirs à enseigner diffusés dans le canton. Deux d'entre eux, le manuel de Vignier (1916) et Maîtrise du français (1979), sont des ouvrages intercantonaux, commandités par plusieurs cantons de Suisse romande. Toutefois ils ont été élaborés dans des conditions différentes : le premier est issu d'un concours suite à la décision des chefs de l'Instruction publique de la Suisse romande d'élaborer le premier moyen d'enseignement commun ; le deuxième a été conçu alors qu'une étape supplémentaire, l'harmonisation de l'enseignement (marquée notamment par un 
programme d'enseignement commun à tous les cantons francophones), était franchie en Suisse romande. Le deuxième manuel de notre corpus, conçu par Albert Atzenwiler - directeur de l'enseignement primaire du canton de Genève, fervent défenseur de l'École active, élève de Charles Bally et considéré comme le « père » de la grammaire genevoise (Bronckart, 1983), est cantonal.

Les manuels soumis à l'analyse sont destinés au degré moyen de l'enseignement primaire. Ils présentent toutefois des caractéristiques spécifiques : le manuel de Vignier (1916) est en réalité un cours de langue et traite conjointement l'enseignement de la grammaire, du vocabulaire et de la composition; celui d'Atzenwiler (1933) est spécifiquement consacré à la grammaire ; enfin, Maîtrise du français (1979) est un ouvrage-cadre destiné à l'enseignant et consacré à l'enseignement de la langue maternelle dans son ensemble.

\subsubsection{Méthodologie d'analyse}

Des critères spécifiques ont été dégagés pour chaque partie des ouvrages (préface, table des matières, chapitres). Sans donner ici le détail des critères mobilisés au cours de l'analyse, nous en esquisserons rapidement les contours. Tout d'abord, les préfaces ont fait l'objet d'une attention particulière consistant à dégager les finalités poursuivies par l'enseignement grammatical, ainsi que la représentation de la langue à l'origine des descriptions grammaticales, et également les éventuelles indications pédagogiques. Cette première étape de l'analyse s'est révélée particulièrement éclairante, dans la mesure où elle nous a permis d'élaborer une vision d'ensemble des principes à l'origine du manuel et de sa structure générale, les auteurs donnant généralement de nombreuses indications quant à l'organisation de l'ouvrage. De fait, nous avons pu dégager un certain nombre d'aspects à investiguer et les mobiliser pour affiner les critères d'analyse des autres parties des ouvrages. Concernant ces dernières, une analyse fine des tables des matières a été menée afin de faire émerger la structuration générale de l'appareil notionnel : les notions sélectionnées et la terminologie utilisée ont tout d'abord été relevées, puis nous avons cherché dans un deuxième temps à comprendre la logique de regroupement et de hiérarchisation de ces différents éléments. Afin de délimiter les critères sous-tendant ces notions, cette étape de l'analyse s'est accompagnée d'un va-et-vient permanent avec l'étude des chapitres. Cette dernière nous a par ailleurs permis de dégager également la démarche d'enseignement préconisée.

\section{Résultats : principaux aspects théoriques et didactiques du corpus de manuels}

\subsection{Unité de l'analyse grammaticale : finalité et fondement épistémologiques}

Les deux premières grammaires de notre corpus (Vignier, 1916; Atzenwiler, 1933) s'appuient sur la proposition comme cadre d'analyse grammaticale. Comme nous le verrons par la suite, des différences notables apparaissent au niveau du regroupement et de l'articulation des notions, mais les deux manuels débutent par une définition de la proposition (notons toutefois que J'apprends la grammaire compte un chapitre d'introduction consacré à différents éléments généraux sur la langue) et s'achèvent par une ouverture sur la notion de phrase. La définition initiale de la proposition est liminaire chez Vignier puisqu'elle se borne à l'ensemble sujet/verbe, mais sans mentionner ces termes techniques ${ }^{4}$ : «Quand on parle d'un être, et qu'on dit ce qu'il fait, on formule une proposition » (p. 6). Du point de vue de la structure, la définition est semblable chez Atzenwiler : «Une proposition est formée au moins d'un sujet et d'un verbe» (p. 29). Toutefois, cette dernière formulation donne une idée plus ouverte de la proposition et laisse entendre qu'elle peut être étendue à l'aide d'autres éléments. De plus, le chapitre consacré à la proposition dans le manuel de 1933 compte les principales fonctions liées au verbe (sujet, attribut, complément d'objet direct, complément d'objet indirect, compléments de lieu, de temps et de manière), ce qui atteste d'une représentation plus large de la proposition.

L'ensemble des notions sélectionnées relève strictement du cadre de la proposition chez Vignier (1916), qui aborde brièvement la notion de phrase dans le dernier chapitre mais annonce explicitement au début 
de l'ouvrage que l'étude de la phrase complexe sera réservée au tome II, soit aux élèves du degré supérieur. La notion de phrase fait en revanche l'objet d'un chapitre chez Atzenwiler (1933), même si son traitement reste relativement restreint. Signe que cette grammaire dépasse le cadre strict de la proposition, le chapitre consacré aux déterminatifs et pronoms intègre la notion de pronom relatif. Plus généralement, et cela concerne les deux manuels, la grande partie des notions sélectionnées relève du niveau de la proposition (parties du discours et fonctions), même si la présence de certaines notions pose question : la présence des temps verbaux, ou encore les notions de style direct et indirect intégrées au chapitre de la phrase dans le manuel d'Atzenwiler dénotent, selon nous, un certain amalgame des niveaux d'analyse (phrase/texte).

Ce cadre d'analyse s'explique en partie par une représentation de la langue relativement similaire chez les deux auteurs. Vignier (1916) justifie les bornes de sa grammaire, limitée à la proposition simple, par une relation directe entre pensée et propriété de la langue: "Toute pensée s'exprimant à l'aide de propositions, nous avons donné comme limites à notre ouvrage l'étude de la proposition simple [...]» (p. VIII). Cette conception de la langue est à première vue similaire chez Atzenwiler (1933), même si le lien pensée/proposition est moins explicite :

\section{Il faut que l'enfant soit constamment et insensiblement entraîné à assembler de la façon la plus juste et la plus claire les signes dont il a besoin pour exprimer sa pensée. Il faut, par des exercices incessants et progressifs, assouplir et affirmer ses procédés d'expression. (Atzenwiler, 1933, p. IV)}

Toutefois, plus loin dans la préface, l'auteur précise, citations de l'ouvrage de Charles Bally $(1930)^{5}$ à l'appui, la relation qui lie selon lui pensée et langue et qui a présidé à la conception de certains des exercices d'application de son manuel : «Il y a de la grammaire dans les mots parce que ceux-ci ne sont jamais isolés dans notre esprit. [...]. Chaque mot est une possibilité illimitée de groupements syntaxiques, comme un concept représente une infinité de jugements en puissance » (Bally, 1930, cité par Atzenwiler, 1933, p. VI). Le parallélisme effectué entre le fonctionnement de la pensée et le fonctionnement syntaxique est particulièrement intéressant, premièrement parce qu'il vient confirmer ce qui était esquissé dans la première citation, soit une conception de la langue comme représentation de la pensée, et deuxièmement parce qu'il laisse entrevoir, selon nous, le principe fondamental de cette grammaire, à savoir une vision profondément sémantique du fonctionnement grammatical. Pour aller plus loin, il nous semble en l'occurrence que les rapports entre discipline de référence (ici, la linguistique) et discipline scolaire se dessinent de manière assez nette, Atzenwiler s'appuyant régulièrement dans sa préface sur des concepts élaborés par Bally et Brunot pour expliciter et justifier la démarche proposée dans son manuel.

Si cette représentation de la langue pose le cadre de l'enseignement grammatical, elle en dessine aussi les finalités : l'enseignement de la grammaire doit être au service de l'expression de la pensée de l'élève. C'est donc une conception profondément représentationnelle de l'enseignement de la grammaire qui est au cour de ces deux manuels. Cette conception a en fin de compte une visée utilitaire : la maîtrise de la lecture et surtout de l'écriture correcte, l'orthographe au sens large du terme. Comme nous le verrons avec le dernier ouvrage de notre corpus, les propositions de renouvellement de l'enseignement de la langue maternelle des années 1970 confèrent également un caractère fortement utilitaire à l'enseignement grammatical. Toutefois, ces propositions mettent l'accent sur le développement de la communication, les conceptions de la langue qui les sous-tendent ayant dépassé celle de langue comme représentation de la pensée.

Chez Atzenwiler (1933), la finalité normative est fortement mise en exergue :

Chaque individu est tenu, sous peine de sanctions de divers ordres, d'utiliser avec un minimum de correction le système de signes de la langue. Il existe une contrainte d'ordre linguistique à laquelle il est impossible d'échapper, même sous prétexte de logique ou d'expressivité. Quoi que l'on fasse, il y a des formes justes et des formes fausses, des constructions correctes et des phrases incorrectes. (p. III)

Nous n'avons pas relevé de prise de position aussi claire dans la grammaire de Vignier (1916), cependant l'incitation à exposer les élèves aux textes des «maîtres de la langue » (p. XII) afin de « former le goût 
[...] et de fournir à leur pensée des modes d'expression» (p. XII) indique clairement que le français littéraire sert de langue de référence. D'autres éléments du manuel viennent confirmer cette hypothèse. Nous en donnerons un exemple tiré de la partie «éléments» de l'ouvrage (voir infra 3.2). Il est alors intéressant de constater que parmi les cinq temps étudiés dans cette partie, faisant office d'introduction à des notions élémentaires (le nom, l'article, l'adjectif, le pronom, le verbe et le temps verbal), figure le passé simple, soumis à des contraintes contextuelles et énonciatives fortes qui restreignent son utilisation (Barceló \& Bres, 2006) et en font le temps par excellence de la narration écrite.

On retrouve ici certaines caractéristiques du modèle classique (Schneuwly \& Dolz, 2009) de l'enseignement de la langue maternelle (notamment la langue comme représentation de la pensée et un amalgame entre langue et grammaire). Ce dernier point est particulièrement visible chez Vignier (1916) dans la mesure où son cours de langue, qui intègre grammaire, vocabulaire et composition est organisé autour d'une progression grammaticale dans laquelle sont insérées des activités de vocabulaire et de composition. Les consignes données au maître pour l'enseignement de la composition sont assez révélatrices, selon nous, de la place centrale de l'enseignement grammatical dans le développement de l'expression : les élèves doivent d'abord s'exercer à « exprimer clairement et succinctement leurs idées » (p. XI) dans un «style bref, coupé, brisé » (p. XI). Peu importe si les transitions sont absentes, c'est par l'étude des textes qu'ils apprendront peu à peu à relier différentes idées entre elles et à structurer leurs textes. L'idée ici sous-jacente, c'est que le développement de l'expression s'appuie en premier lieu sur la production de phrases correctes, que l'élève apprend peu à peu à lier entre elles afin de produire un texte.

Il est intéressant de constater que, par comparaison, les prétentions affichées dans la préface de Maîtrise $d u$ français (1979) dénotent une approche radicalement nouvelle de l'enseignement de la langue maternelle, qui doit être, selon les auteurs, au service de la communication :

Faire en sorte que l'enfant maîtrise sa langue maternelle, que le français devienne pour lui un véritable moyen de communication et de libération, quelle noble ambition, quel ferment de motivation profonde ! (Besson, Genoud, Lipp \& Nussbaum, 1979, p. $\mathrm{XV})$

Cette prise de position permet de justifier des modifications en apparence importantes du découpage disciplinaire et de la fonction qu'y occupe l'enseignement grammatical. L'enseignement de la langue maternelle dans son ensemble est appréhendé comme un moyen pour l'élève de développer ses capacités de communication. Dans cette configuration, la grammaire est envisagée comme un outil, au même titre que d'autres, qui doit servir cette finalité. Ces outils (syntaxe, lexique, lecture, élocution, orthographe) prennent la forme d'ateliers intégrés dans des activités-cadres définies par des objectifs langagiers. Un autre aspect important de cet ouvrage est la conception de la langue qui «est à la fois liberté et contraintes » (Besson \& al, 1979, p. 4). La langue permet donc de s'exprimer mais comme elle est également un code (la définition qui en est donnée dénote l'influence des théories linguistiques, notamment de la conception saussurienne de la langue), un certain nombre de règles doivent être respectées pour permettre la communication entre locuteurs.

Par ailleurs, la prise en compte de facteurs sociologiques et psychologiques entraîne une conception nouvelle de l'enfant. En effet, l'apprentissage de la langue maternelle n'est pas, pour les auteurs, le seul fait de l'école, l'élève ayant déjà une certaine maîtrise de la langue à son entrée dans le système scolaire. En outre, la variation des niveaux de langue est prise en compte. L'enseignement de la langue doit permettre à l'élève d'adapter son langage à diverses situations de communication.

Concernant plus particulièrement l'enseignement grammatical, les différentes notions qui relevaient de l'ensemble "grammaire» dans les manuels précédents sont ici réparties entre plusieurs ateliers (orthographe, syntaxe, morphosyntaxe du verbe et lexique) : l'orthographe constitue une composante spécifique, disjointe de l'enseignement syntaxique ; la morphologie nominale, rattachée à l'enseignement grammatical dans le manuel d'Atzenwiler (1933) est ici intégrée à l'atelier de lexique ; enfin, les notions de temps verbaux et de mode sont détachées de la syntaxe et traitées dans une partie consacrée à la morphosyntaxe du verbe. Le chapitre traitant des phénomènes syntaxiques est structuré autour de la phrase P qui sert d'unité d'analyse. 
Maîtrise du français vise une approche descriptive des faits de langue. En effet, la phrase est saisie à partir de son fonctionnement syntaxique, contrairement à la proposition, qui est définie précisément comme unité de pensée. La définition de la phrase exposée dans la partie "Introduction théorique » de l'ouvrage illustre bien, en comparaison avec les définitions de la proposition relevées dans les deux autres manuels (voir précédemment), le renouvellement épistémologique à l'œuvre dans les années 1970 :

Nous optons pour une décomposition de la phrase déclarative en deux groupes d'éléments : le groupe nominal et le groupe verbal. [...]

En structure profonde, le groupe nominal qui précède le groupe verbal est constituant obligatoire de la phrase. On dit qu'il est le sujet de la phrase.

Le groupe verbal est aussi constituant obligatoire de la phrase. On dit qu'il est le prédicat de la phrase. Le groupe verbal comprend un verbe qui entretient un rapport formel avec le groupe nominal sujet. (Besson \& al, 1979, p. 341)

\subsection{Notions sélectionnées et progression}

Comme nous l'avons vu précédemment, la proposition sert de cadre au manuel de Vignier (1916) et elle en régit la progression. Le manuel est organisé en cinq parties, chacune traitant un aspect spécifique de la proposition : la première donne une définition de la proposition autour de l'ensemble sujet/verbe, puis viennent ce que Vignier appelle les « éléments »; suivent ensuite les « termes » de la proposition et enfin les «formes ». La dernière partie constitue une étude complémentaire des éléments de la proposition. Cette partie est à destination des enseignants qui avanceraient plus vite ou consacreraient davantage de temps à l'enseignement grammatical. Comme l'auteur l'annonce en préface, cet ouvrage délaisse l'ordre traditionnel des parties du discours dans lequel sont, à l'époque, le plus souvent présentés les faits grammaticaux. Plus intéressant encore est l'explication que donne l'auteur pour justifier ce choix :

En grammaire, nous avons abandonné délibérément l'ordre traditionnel dans lequel on avait coutume d'étudier les parties du discours: les faits grammaticaux s'enchaînent entre eux, et la place de leur étude est marquée par cet enchaînement même. (Vignier, 1916, p. VIII)

Il y aurait donc, selon Vignier, un ordre «naturel» des faits de langue qui s'imposerait de fait. Or, d'autres facteurs semblent présider à l'organisation de cet ouvrage : le rythme scolaire et le découpage disciplinaire (l'année scolaire compte un nombre déterminé d'heures d'enseignement qui sont réparties entre les différentes disciplines, cette distribution étant définie par les programmes). L'ouvrage est effectivement pensé pour être utilisé dans l'ordre, leçon après leçon, semaine après semaine. Le nombre de leçons a même été calculé en fonction du nombre de semaines que compte l'année scolaire. La progression proposée à l'enseignant est très fine, les différentes leçons peuvent, et doivent même être étudiées dans l'ordre proposé puisqu'elles s'enchaînent les unes aux autres, comme l'illustre l'extrait de la table des matières du manuel retranscrit ci-après : 
Figure 1 : Extrait de la table des matières de Cours de langue française (Vol. 1) (Vignier, 1916)

\begin{tabular}{|c|c|}
\hline 2. LES ÉLÉMENTS DE LA PROPOSITION. & \\
\hline Le $n o m \ldots \ldots \ldots \ldots \ldots \ldots \ldots \ldots \ldots \ldots \ldots$ & 10 \\
\hline Le nombre du nom.................. & 15 \\
\hline Le pluriel du nom.... & 19 \\
\hline Le genre du nom......... & 24 \\
\hline L'article ; l'apostrophe............... & 28 \\
\hline L'adjectif qualificatif... & 32 \\
\hline L'accord de l'adjectif en genre.... & 37 \\
\hline L'accord en nombre...... & 41 \\
\hline Le verbe....... & 46 \\
\hline Première idée du pronom............. & 51 \\
\hline La personne ; le pronom personnel............ & 56 \\
\hline L'accord du verbe............. & 60 \\
\hline Les trois temps du verbe............ & 64 \\
\hline Le présent ; radical et terminaison............. & 68 \\
\hline Les verbes être et avoir..... & 73 \\
\hline Un passé : l’imparfait............. & 77 \\
\hline Un $2^{\text {me }}$ passé : le passé simple....... & 82 \\
\hline Un $3^{\text {me }}$ passé : le passé composé... & 86 \\
\hline Un futur : le futur simple..... & 91 \\
\hline Révision des chap. 1 et 2 . & 94 \\
\hline
\end{tabular}

Afin d'étayer notre propos, prenons l'exemple du verbe : comme nous pouvons le voir sur la figure cidessus, une leçon initiale est consacrée à l'accord du verbe en nombre (leçon intitulée « le verbe »), puis une deuxième leçon aborde l'accord en nombre et en personne (leçon intitulée " l'accord du verbe »), enfin une troisième introduit les trois temps du verbe (passé, présent, futur). Ce découpage, qui peut paraître surprenant au premier abord, suit une logique que l'on comprend dès lors que l'on s'intéresse de près à la structuration générale de l'intégralité de la partie introduisant les «éléments ». En effet, la première leçon traitant de l'accord en nombre suit un ensemble de leçons consacrées entre autres à l'accord du nom et de l'adjectif. L'élève est donc, à ce stade, familiarisé avec la notion de nombre. Dans la même logique, la seconde leçon sur le verbe, consacrée à l'accord en nombre et en personne, succède directement à la définition du pronom et à l'introduction de la notion de personne. Sur cette base, dès lors que l'élève a identifié que la terminaison du verbe variait en fonction du nombre et de la personne, est introduite la notion de variation liée au temps verbal. Les notions ont donc été découpées et regroupées selon une logique de progression " linéaire " (on passe d'un objet à un autre en fonction des " affinités " entre ces objets). Nous voyons donc ici, en acte, le passage d'une organisation en fonction de la logique d'analyse de l'objet à une organisation en fonction de l'élève. 
Notons que le découpage de la proposition en « éléments », «termes » et «formes » pose problème à première vue. Il semblerait en effet que les «éléments» correspondent aux parties du discours et les « termes » aux fonctions, or cela ne résiste pas à l'analyse. Les " termes » sont composés de notions disparates : on y trouve des fonctions (sujet, objet, compléments circonstanciels, etc.) mais également des éléments traditionnellement classés dans les parties du discours (préposition et adverbe). Pour l'adverbe, cela s'explique dans la mesure où il est défini d'un point de vue fonctionnel - il « suffit à lui seul pour marquer le temps, le lieu, la manière, la quantité » (Vignier, 1916, p. 117) - mais ce n'est absolument pas le cas de la préposition qui est définie du point de vue de son fonctionnement syntaxique. Or, ce découpage s'explique par la dimension profondément sémantique de cette grammaire, attestée notamment par des définitions majoritairement référentielles (Ex: «Les mots qui indiquent ce que font les personnes, les animaux et les choses sont des verbes » (Vignier, 1916, p. 2) ; «Le mot qui désigne une personne, un animal ou une chose est un nom » (p. 10)) et les procédés mobilisés pour identifier les compléments (utilisation des mots interrogatifs qui, que, à qui, de qui, quand, où, comment, etc.). Enfin, l'analyse de l'appareil notionnel de ce manuel révèle également la quasi-disparition des notions issues de la grammaire scolaire du XIXe siècle ${ }^{6}$.

La grammaire d'Atzenwiler (1933) suit une logique différente : les notions sont regroupées en chapitres autonomes les uns par rapport aux autres et présentant une certaine unité. Concernant la présentation des parties du discours, des divergences notables avec l'ordre dans lequel elles sont traditionnellement regroupées apparaissent : les pronoms et les déterminatifs sont traités dans un même chapitre, afin de mettre en parallèle le fonctionnement des uns et des autres (les adjectifs et les pronoms démonstratifs sont étudiés dans la même leçon, idem pour les adjectifs et pronoms possessifs, etc.) ; un chapitre est consacré aux mots invariables comprenant adverbe, préposition, conjonctions et interjections; tout ce qui concerne le verbe est regroupé dans un même chapitre à l'allure en conséquence un peu hétéroclite (conjugaison, mode, accord des participes, formation des verbes, forme passive, etc.). Mais cette grammaire présente surtout une innovation d'importance: l'article et les adjectifs démonstratifs, possessifs, indéfinis, numéraux et interrogatifs sont regroupés dans une même classe appelée « déterminatifs ».

Les critères sous-tendant les définitions grammaticales sont le plus souvent sémantiques, comme chez Vignier (1916), mais d'autres critères sont également mobilisés pour certaines notions difficilement appréhensibles à partir de critères sémantiques. C'est le cas par exemple de la préposition, de la conjonction, des déterminatifs. De même, le procédé proposé aux élèves pour identifier les différents compléments de la proposition est identique à celui relevé dans le cours de Vignier (utilisation des questions : qui est-ce que? qui est-ce qui? où? quand? comment? etc.). Il nous semble ici que l'influence de Charles Bally et de Ferdinand Brunot, auxquels Atzenwiler (1933) se réfère dans la préface est patente. En effet, si des désaccords conceptuels ont pu opposer les deux linguistes (voir à ce propos Chevalier, 1990), leurs travaux révèlent néanmoins, comme le montre Chiss (1995), des préoccupations communes qui semblent avoir nourri la réflexion d'Atzenwiler et orienté sa démarche : nous donnerons pour exemples la critique des règles données a priori, l'importance donnée à la démarche inductive, l'enseignement de la grammaire au service du développement de l'esprit. Ce cadre de référence explique en partie l'orientation sémantique de cette grammaire mais il influence surtout sur la démarche d'enseignement, comme nous le verrons par la suite.

Maîtrise du français (1979) marque, du point de vue des notions sélectionnées, une véritable rupture. La partie syntaxe, à laquelle nous nous intéresserons plus particulièrement ici, est structurée autour de deux démarches : la description des faits de langue d'une part, l'explication du fonctionnement du langage d'autre part. En effet, l'enseignement grammatical doit, selon les auteurs, permettre aux élèves de comprendre l'organisation et le fonctionnement du langage. Deux théories de référence sont mobilisées : la grammaire distributionnelle et la grammaire générative transformationelle. La description du fonctionnement de la langue est donc basée sur des critères uniquement syntaxiques. Prenons comme exemple des extraits de l'introduction théorique consacrés à la notion de groupe nominal sujet :

En structure profonde, le groupe nominal qui précède le groupe verbal est constituant obligatoire de la phrase. On dit qu'il est le sujet de la phrase.

$[\ldots]$ 


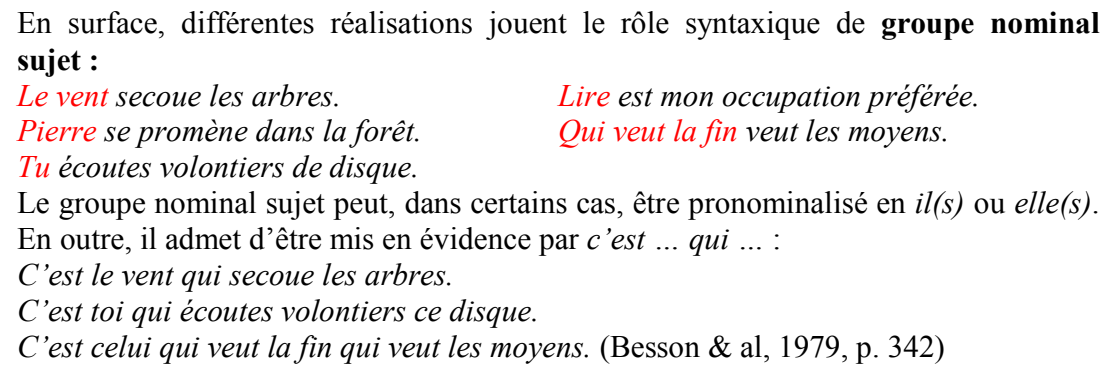

Il apparait clairement dans cet extrait que la dimension sémantique est totalement absente : le groupe nominal sujet est avant tout défini par son caractère obligatoire et sa distribution syntaxique (il précède le groupe verbal). L'influence de la grammaire générative est notable dans la distinction des différentes structures (profonde/de surface). Enfin, la mobilisation des procédés distributionnalistes d'extraction et de substitution parachève la définition première, donnant une idée complète du fonctionnement syntaxique du groupe sujet. Cette démarche se démarque ainsi des grammaires précédentes, dans lesquelles le fonctionnement syntaxique est saisi dans sa relation avec l'expression des idées.

Par ailleurs, au contraire des deux premiers manuels du corpus, Maîtrise du français (1979) ne procède pas à un traitement exhaustif de toutes les fonctions et catégories grammaticales. La partie théorique est centrée sur la phrase : partant du niveau phrastique (types de phrase, définis d'un point de vue énonciatif), elle en traite ensuite les constituants (groupe nominal, groupe verbal, groupes facultatifs) pour se focaliser à nouveau sur la phrase (phrases enchâssées). On propose aux élèves d'adopter une démarche de linguiste : ces derniers sont en effet amenés à faire de nombreuses manipulations pour « démonter les principaux mécanismes de la langue et en inventorier les possibilités » (Besson \& al, 1979, p. 337).

\subsection{Démarche d'enseignement}

Les démarches d'enseignement adoptées dans les manuels de Vignier (1916) et d'Atzenwiler (1933) sont de type inductif. Critiquant avec force la méthode déductive consistant à appliquer des règles apprises par cœur, les deux auteurs insistent particulièrement sur la nécessité d'amener l'élève à comprendre le fonctionnement des faits grammaticaux à partir de leur observation. A première vue similaires, les deux démarches proposées présentent néanmoins certaines différences majeures sur lesquelles nous reviendrons par la suite. Elle est structurée en trois étapes chez Vignier, quatre chez Atzenwiler, que nous avons schématisées de la manière suivante :

1. Activité de production orale (Atzenwiler)

2. Observation du fonctionnement de la notion étudiée (Vignier/Atzenwiler)

3. Enonciation de la règle (Vignier/Atzenwiler)

4. Application de la règle à travers divers exercices (Vignier/Atzenwiler)

Ce schéma révèle une première différence notable entre les démarches préconisées dans les deux manuels. En effet, bien que les observations grammaticales soient menées à partir d'un texte-support dans les deux grammaires, cette étude est systématiquement précédée d'une activité orale dans l'ouvrage d'Atzenwiler (1933) : les élèves sont invités à produire des énoncés oraux (dialogues, descriptions, etc.) à partir de situations de communication ancrées dans leur quotidien. En guise d'exemple, voici la consigne donnée en ouverture du chapitre sur le complément d'objet direct :

\section{Cherchons des exemples.}

a) C'est la récréation du matin; dites ce que les élèves mangent pour leurs dix heures.

b) Dites ce qu'on voit de la fenêtre de la classe. (Atzenwiler, 1933, p. 40)

Cet exemple montre l'émergence, dès 1933, de l'activité langagière comme point de départ à toute réflexion sur le fonctionnement de la langue. Il nous semble également assister à l'esquisse d'un 
décloisonnement de l'enseignement de la langue maternelle, thème cher à Bally (Chiss, 1995). Ce décloisonnement est déjà amorcé chez Vignier, dont le cours de langue joint enseignement de la composition et du vocabulaire à celui de la grammaire, mais il revêt ici un caractère novateur par la prise en compte de l'activité orale.

Par ailleurs, la démarche inductive consiste à permettre à l'élève de comprendre par lui-même le fonctionnement des faits de langue, cela à partir de l'observation guidée d'un texte. Cette activité d'observation est menée de manière différente dans les deux manuels. Prenons pour exemple celle proposée par chaque auteur sur l'adjectif:

\section{Observations.}

L'auteur, qui veut décrire la voiture, parle de sa couleur (elle est jaune), de la couleur des roues (devenues grises à l'usage), de leurs dimensions (les unes petites, les autres hautes). Il dit plus loin la forme des paniers (ils sont ronds). [...]. (Vignier, 1916, p. 32)

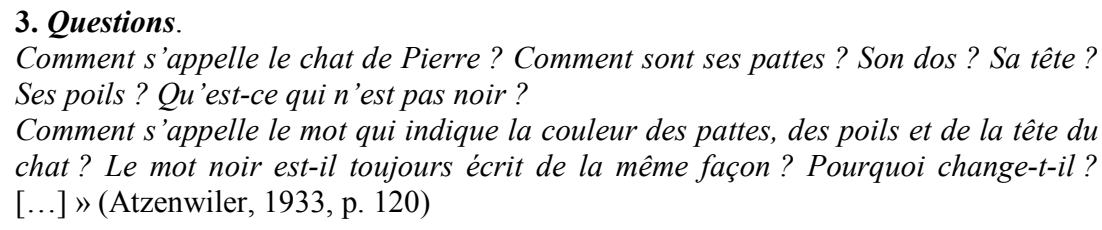

Les deux extraits ci-dessus illustrent bien une divergence dans les démarches d'observations élaborées par les deux auteurs. Celle préconisée par Atzenwiler (1933), si elle oriente par des questions l'attention de l'élève sur les différents aspects de l'adjectif afin de structurer ses observations, lui permet au demeurant de réfléchir au fonctionnement de cette notion. En revanche, la démarche préconisée par Vignier (1916) est de type directif et permet plus difficilement à l'élève de mener une réflexion personnelle. Toutefois, Vignier précise bien, dans les « indications pédagogiques » faisant suite à la préface, que ces observations «n'ont en rien la prétention de remplacer la leçon du maître : elles seront pour celui-ci un guide utile, elles pourront rendre à l'élève le service de mémento » (p. X).

Concernant les textes supports à partir desquels se font les observations grammaticales, il s'agit chez Vignier (1916) de textes littéraires, tirés des « meilleurs auteurs des $18^{\mathrm{me}}, 19^{\mathrm{me}}$ et $20^{\mathrm{me}}$ siècles » (p. VIII). En voici un exemple, extrait de la leçon sur l'adjectif qualificatif :

« Texte.

LA DiligenCE. - La diligence allait quitter le village. C'était une voiture jaune, montée sur des roues jaunes aussi autrefois, mais rendues grises par l'accumulation des boues. Celles de devant étaient toutes petites ; celles de derrière, hautes et frêles, portaient le coffre. Trois rosses blanches attelées en arbalète devaient traîner cette carriole.

Le cocher, un petit homme à gros ventre, la face rougie par le grand air des champs, apparut sur la porte de l'hôtel en s'essuyant la bouche d'un revers de main. De larges paniers ronds, pleins de volailles effarées, attendaient devant des paysans immobiles.

Guy de MAUPASSANT » (Vignier, 1916, p. 32)

Atzenwiler (1933) privilégie des textes qui se rapprochent, selon lui, le plus possible du langage des élèves et de leurs préoccupations (travaux d'élèves, textes littéraires choisis pour leur «style concret»). Par conséquent, les textes sélectionnés dans ce manuel marquent un contraste assez saisissant avec les supports privilégiés par Vignier, comme l'illustre bien cet extrait, issu de la leçon consacrée à l'adjectif qualificatif :

\section{«2. Le chat de Pierre.}

Le chat de Pierre s'appelle Misou.

Il a le dos noir, la tête noire, les pattes noires, bref, tous les poils noirs : c'est un vrai morceau de charbon.

Seuls ses yeux verts et sa langue rose éclairent cette touffe sombre. » (Atzenwiler, 1933, p. 120) 
Il semble donc que le français littéraire serve fortement de langue de référence dans le cours de Vignier (1916), tandis que cette norme apparaît comme moins présente dans l'ouvrage d'Atzenwiler (1933).

Maîtrise du français radicalise l'approche inductive déjà présente dans les grammaires précédentes. Les auteurs distinguent deux domaines de l'enseignement : celui de la libération de la parole dans lequel se pratiquent les activités langagières (parler, écouter, lire écrire) dans des projets de classe (monter une exposition par exemple) appelés «activités cadres»; et celui de la structuration de la langue dont l'institution didactique principale est l'atelier. Ce dernier est véritablement conçu comme un lieu de travail sur la langue, la référence de ce travail étant celui du linguiste lui-même.

Le travail en atelier a toujours comme point de départ un corpus : un ensemble de formes linguistiques (phrases, groupes, mots, voire extraits de textes) qui sont soit fournies par l'enseignant, soit créées par les élèves dans des situations de production dirigée. Ce corpus est soumis à une série d'opérations comme des catégorisations ou des manipulations (ajout, dédoublement, déplacement, effacement, remplacement). Prenons l'exemple de l'adjectif, présenté dans l'ouvrage (p. 377).

Travail sur l'expansion du GN

Soit le corpus suivant, élaboré (fictivement) avec les élèves d'une classe :

Maman a acheté une voiture neuve

de course

rapide

qui a peu roulé

à vitesses automatiques

que ma sœur déteste

une petite voiture

Tâches des élèves :

1 repérer les constituants obligatoires par des manipulations d'effacement par exemple

2 essayer de faire un classement des constituants facultatifs : 1) précédés de «qui »;2) précédés de « de », à, ..., 3) qui suivent ou précèdent directement le nom

3 manipulation de déplacement pour montrer que les deux premiers suivent toujours le nom

\section{Identification de l'adjectif}

Un nouveau corpus est élaboré ne comprenant que des expansions de type 3. Tâches des élèves :

a) observation : suit ou précède immédiatement le nom

b) déplacement : peut être souvent déplacé avant ou après le nom

c) ajout : peut être accompagné de « très », « trop », etc.

A travers de tels ateliers, Maîtrise du français vise à permettre aux élèves d'observer et de décrire la langue telle qu'elle fonctionne réellement (au moins dans certaines de ses manifestations surtout écrites). Sa prétention est d'être tendanciellement non normative. Il s'agit pour l'élève de mener un travail de réflexion sur la langue, de la connaître et de disposer d'un appareil conceptuel pour la décrire, plus particulièrement au niveau de la phrase, du mot, de la morphosyntaxe du verbe, de la phonologie. Le travail est basé sur l'intérêt supposé de l'élève pour la langue. Il en découle un choix d'une pédagogie active de recherche et d'aborder la langue comme objet de connaissance. Par une approche inductive, l'élève est amené à découvrir dans une certaine mesure des régularités et catégories de la langue, voire de formuler des règles essentielles de fonctionnement de la langue. L'atelier, institution didactique centrale de travail grammatical dans Maîtrise du français, est le lieu de manipulation de la langue en vue de 
découvrir certaines de ses propriétés et de faire de la langue un objet de réflexion de connaissance, d'observation, et plus généralement même, de construire par rapport à la langue un rapport différent, distancié. L'homologie avec le travail du linguiste - établissement d'un corpus, manipulations et catégorisation - est la démarche proposée pour y parvenir : il y a donc dans Maîtrise du français non seulement transposition didactique de notions, mais aussi de démarches qui permettent de les construire.

\section{Conclusion}

Cette recherche nous a permis de mettre en évidence la transformation progressive de l'enseignement grammatical, tel que pensé dans les manuels scolaires en usage dans le canton de Genève au cours de la période 1916-1979. Les deux premiers manuels de notre corpus présentent une certaine unité au niveau notionnel, de par la dimension profondément sémantique de l'enseignement grammatical qu'ils proposent. Cette dimension est en parfaite concordance avec les références que les deux auteurs mobilisent dans leurs préfaces : les références sont explicites et clairement revendiquées chez Atzenwiler (1933), qui illustre son propos par de nombreuses citations des linguistes Ferdinand Brunot et Charles Bally. La référence est plus allusive mais bien présente chez Vignier (1916) : «En le composant [le Cours de langue française], nous nous sommes inspirés des idées exposées par les propagateurs de la réforme de l'enseignement grammatical en France » (p. VIII). Si aucune mention directe à Ferdinand Brunot n'a été relevée, on peut toutefois supposer qu'il figure parmi les références de Charles Vignier, le linguiste français ayant été l'un des plus ardents défenseurs de la réforme en France et également en contact avec la Suisse romande?

Sur le plan didactique, la première moitié du XXe siècle est marquée par des innovations, au niveau de la démarche d'enseignement notamment. Le manuel de Vignier (1916) présente une démarche profondément inductive, revisitée par Atzenwiler (1933) qui, comme nous l'avons vu, propose une approche novatrice, en initiant les activités grammaticales à partir d'une activité orale. Une analyse des rééditions et adaptations de ces manuels permettrait peut-être de mettre à jour des étapes intermédiaires dans l'évolution observée ici.

Enfin, les années 1970 sont le théâtre d'une transformation majeure sur le plan théorique, comme l'a montré l'analyse de Maitrise du français (1979), avec la mobilisation des théories issues de la linguistique. L'enseignement de la langue maternelle est conçu de manière décloisonnée, les composantes telles que la grammaire devant être au service du développement des moyens de communication de l'élève. La démarche d'enseignement proposée est également inductive, dans la mesure où elle est pensée de façon à permettre aux élèves d'observer et de décrire le fonctionnement de la langue.

Par ailleurs, cette présentation a fait apparaitre un certain nombre de points nécessitant une analyse plus approfondie, qui feront l'objet de recherches ultérieures dont nous esquissons ici les grandes lignes. Tout d'abord, il est prévu d'étendre les bornes chronologiques de notre étude, en incluant à notre corpus les manuels du XIXe siècle. De même, il importera de mener des analyses similaires sur le degré supérieur du primaire, les manuels étant généralement élaborés en séries (un premier volume pour le degré intermédiaire et un second pour le degré supérieur). Ces travaux seront complétés par l'analyse des manuels en usage dans le secondaire I. Enfin, la Suisse présentant la spécificité d'avoir des systèmes éducatifs cantonaux, il est prévu de mener des recherches similaires sur les cantons de Vaud et de Fribourg afin de mener des comparaisons.

Enfin, cette communication est centrée sur le niveau des savoirs à enseigner tels que présentés dans les moyens d'enseignement. Il importera donc d'élargir notre recherche en y intégrant d'autres sources : un certain nombre de documents conservés dans les archives de l'Instruction publique des trois cantons (courriers, rapports de commission des manuels) seront dépouillés afin de mieux cerner les conditions de production des manuels ; l'analyse des programmes d'enseignement nous permettra de confronter les savoirs à enseigner tels que pensés dans les moyens d'enseignement aux savoirs figurant dans les prescriptions officielles; l'étude des débats et controverses ayant marqué l'enseignement de la langue maternelle via l'analyse d'un ensemble de revue pédagogiques est également prévue. 


\section{Bibliographie}

\section{Sources}

Atzenwiler, A. (1933). J'apprends la grammaire... Leçons et exercices de grammaire française (Vol. 1). Genève : Imprimerie P.-E. Grivet.

Besson, M.-J., Genoud, M.-R., Lipp, B. \& Nussbaum, R. (1979). Maîtrise du français. Office romand des éditions et du matériel scolaire.

Vignier, Ch. (avec Briod, U., Jayet, L. \& Sensine, H.). (1916). Cours de langue française. Grammaire, vocabulaire, composition (Vol. 1). Genève : Librairie R. Burkhardt.

\section{Littérature de référence}

Barceló, G.-J. \& Bres, J. (2006). Les temps de l'indicatif en français. Paris : Editions Ophrys.

Bronckart, J.-P. (1983). Réformer l'enseignement du français. Pourquoi? Comment? Education et Recherche, 5, 9-21.

Bronckart, J.-P (2001). Enseigner la grammaire dans le cadre de l'enseignement rénové de la langue (Cahier du Secteur des langues - Français $\mathrm{N}^{\circ} 75$ ). Genève : Département de l'Instruction publique.

Chervel, A. (1977). ... et il fallut apprendre à lire à tous les petits Français. Histoire de la grammaire scolaire. Paris: Payot.

Chervel, A. (2006). Histoire de l'enseignement du français du XVIIe au XXe siècle. Paris: Retz.

Chevalier, J.-C. (1990). Syntaxe et sémantique en grammaire. Histoire d'une méprise : F. Brunot et Ch. Bally. In R. Liver, I. Werlen \& P. Wunderli (Ed.), Sprachtheorie und Theorie der Sprachwissenschaft (pp. 95-107). Tübingen : Narr Verlag.

Chevallard, Y. (1985). La transposition didactique: du savoir savant au savoir enseigné. Grenoble : La pensée sauvage.

Chiss, J.-L (1995). A partir de Bally et Brunot: la langue française, les savants et les pédagogues. Histoire Epistémologie Langage, 17(1), 19-40.

Choppin, A. (1980). L'histoire des manuels scolaires. Une approche globale. Histoire de l'éducation, 9, 1-25.

Darme, A. (2013). Transformation des savoirs scolaires en Suisse romande (1830-1990) : le cas de la grammaire au travers de l'analyse et des prescriptions. Canevas de thèse en Sciences de l'Education, Université de Genève.

Dorier, J.-L., Leutenegger, F. \& Schneuwly, B. (2013). Le didactique, les didactiques, la didactique. In J.-L. Dorier, F., Leutenegger, \& B. Schneuwly, (Eds.). Didactique en construction, constructions des didactiques (pp. 7-35). Bruxelles : De Boeck.

Fournier, J.-M. (1998). La constitution d'une langue de référence de l'enseignement de la grammaire. L'exemple de la nomenclature grammaticale de 1910. Les carnets $d u$ Cediscor, 5, 39-48. Tiré de http://cediscor.revues.org/271.

Hofstetter, R., Schneuwly, B., Lussi, V. \& Haenggeli-Jenni, B. (2006). L'engagement scientifique et réformiste en faveur de la «nouvelle pédagogie». Genève dans le contexte international - premières décades du 20e siècle (pp. 107-142). In R. Hofstetter \& B. Schneuwly (Ed.) Passion, Fusion, Tension. New Education and Educational sciences - Education nouvelle et sciences de l'éducation (end 19th-middle 20th century - fin 19e - milieu 20e siècle). Berne : Lang.

Savatovsky, D. (2011). Une néo-rhétorique de l'école (1985-2005). In J.-L. Chiss, H. Merlin-Kajman \& C. Puech (Ed.), Le français, discipline d'enseignement : histoire, champ et terrain (pp. 123-139). Paris : Riveneuve éd.

Schneuwly, B. (1995). De l'utilité de la transposition didactique. In J.-L Chiss, J. David \& Y. Reuter (Ed.), Didactique du français. Etat d'une discipline (pp. 47-62). Paris: Nathan.

Schneuwly, B. \& Dolz, J. (2009). Des objets enseignés en classe de français. Rennes : PUR.

\footnotetext{
${ }^{1}$ Charles Bally a donné en 1930 un cycle de cinq conférences à l’Université de Genève, consignées dans l'ouvrage suivant : Bally, Ch. (1930). La crise du français. Notre langue maternelle à l'école. Neuchâtel : Delachaux et Niestlé. Dans cet ouvrage, l'auteur développe sa conception de la langue maternelle et avance des propositions en matière de didactique du français résolument novatrices pour l'époque.
} 
${ }^{2}$ Elaboré dans le cadre du projet de recherche Sinergia « Transformation du savoir scolaire depuis 1830 » $\left(\mathrm{N}^{\circ}\right.$ CRSII1_141826), la présente communication se base sur une recherche en cours, effectuée dans le cadre d'un doctorat s'intéressant à la transformation des savoirs scolaires grammaticaux en Suisse romande au cours de la période 1830-1990 (Darme, 2013). De ce fait, et de par la spécificité du projet-cadre dans lequel cette recherche s'insère' l'analyse présentée ici sera l'objet de développements ultérieurs (extension de la période de recherche et comparaisons intercantonales, interrégionales - allemand, français et italien en Suisse - voire internationales).

${ }^{3}$ Département de l'Instruction Publique du canton de Genève (DIP). (1949). Leçons et exercices de grammaire française (Vol. 1). Genève : Impr. P.E. Grivet.

${ }^{4}$ Notre recherche adoptant un point de vue historique, le choix de la terminologie grammaticale utilisée tout au long de l'analyse sera fonction de celle relevée dans les manuels analysés.

${ }^{5}$ Voir note 1.

${ }^{6}$ Concernant ce point, une rapide incursion dans la grammaire en usage dans le canton de Genève au tout début du XXe siècle, soit juste avant le Cours de langue de 1916 - Vignier, Ch. (1908). Cours élémentaire et pratique de grammaire française à l'usage des écoles primaires suivi d'un vocabulaire. Premier volume. Genève: Imprimerie W. Kündig \& Fils - se montre particulièrement révélatrice des changements que connait cette période en matière d'enseignement grammatical. En effet, cette grammaire, également élaborée par Charles Vignier, et ne précédant que de huit ans le Cours de langue, contient encore un certain nombre de notions héritées de la première grammaire scolaire (Chervel, 1977). Parmi celles-ci, on donnera en exemple la présence des quatre conjugaisons (-er, -ir, -oir, re), ou encore de la distinction temps primitifs/temps dérivés. Ces notions sont complètement absentes de la grammaire de 1916.

${ }^{7}$ Ferdinand Brunot fut invité au congrès des enseignants romands en 1910 et cité comme référence principale par Claparède et l'institut Jean-Jacques Rousseau. 